\title{
LA PERMISIBILIDAD ÉTICA DE LAS TECNOLOGÍAS DE BIOMEJORA MORAL*
}

\author{
ANÍBAL MONASTERIO ASTOBIZA \\ Universidad del País Vasco
}

\begin{abstract}
RESUMEN: Los recientes avances en neurociencia, y en las ciencias de la vida en general, han hecho posible no solo tratar y restaurar las funciones dañadas, sino la posibilidad de algún día mejorar las funciones en la población saludable. Las amplias aplicaciones de la biotecnología y medicación farmacológica se dirigen a los mecanismos próximos que se consideran deficitarios en los pacientes. Recientemente, se ha mostrado como algunas de estas medicaciones pueden afectar comportamientos complejos humanos, como la moral en personas saludables (Crockett et al. 2015), lo cual abre la puerta para un entendimiento más profundo de la naturaleza humana. ¿Hasta que punto es éticamente permisible alterar la neuroquímica o modular una región cerebral para intervenir en el comportamiento moral?, ¿es intrínsecamente malo la biomejora moral?, ¿cómo podemos conocer los efectos adversos y cómo sopesarlos en un análisis coste/beneficio de una intervención?, ¿la biomejora moral supone una amenaza a la libertad? Considerando dos posturas opuestas, DeGrazia (2014) y Harris (2011), sobre los retos éticos de la biomejora moral se sugiere que si los principios tradicionales de la bioética como la seguridad, efectividad y universalidad se cumplen no hay nada malo en la biomejora moral per se y, por consiguiente, es éticamente permisible.
\end{abstract}

PALABRAS CLAVE: biomejora moral; libertad; permisibilidad ética.

\section{The ethical permissibility of moral bioenhancement technologies}

ABSTRACT: The recent advances in neuroscience, and the life sciences in general, have made possible not only to treat and restore damaged functions but someday may allow us to improve and enhance functions in healthy people. The wide range of applications of biotechnology and pharmacology target the proximate mechanisms that are thought to be impaired in the clinical population. Recently, it has been shown that some of these medications could affect complex human behavior, such as morality in healthy people (Crockett et al. 2015), which open the window to a deeper understanding of human nature. To what extent is ethically permissible to alter brain neurochemistry or modulate some brain region to intervene in moral behavior? Is moral bioenhancement intrinsically wrong? How can we know the undesirable side-effects and how to weighing them in a cost/benefit analysis of a given intervention? Does moral bioenhancement pose a threat to human freedom? Considering two polar opposites, DeGrazia (2014) and Harris (2011), on the ethical challenges of moral bioenhancement

* Agradezco el patrocinio del Gobierno Vasco para desarrollar una beca posdoctoral de investigación en el Uehiro Centre for Practical Ethics de la Universidad de Oxford y a esta última institución su cálida acogida. Este trabajo se ha realizado en el marco del proyecto de investigación KONTUZ!: «Responsabilidad causal de la comisión por omisión: Una dilucidación ético-jurídica de los problemas de la inacción indebida» (MINECO FFI201453926-R); el proyecto de investigación: "La constitución del sujeto en la interacción social: identidad, normas y sentido de la acción desde la perspectiva de la filosofía de la acción, la epistemología y la filosofía experimental" (FFI2015-67569-C2-2-P)»; y el proyecto de investigación "Artificial Intelligence and Biotechnology of Moral Enhancement. Ethical Aspects»(FFI2016-79000-P)». E-mail personal: anibalmastobiza@gmail.com 
is suggested that if traditional bioethical principles of safety, effectiveness and universality are met there's nothing wrong with moral bioenhancement per se and therefore is ethically permissible.

KEY WORDS: moral enhancement; freedom; ethical permissibility.

\section{INTRODUCCIÓN}

El creciente desarrollo del conocimiento neurocientífico y biomédico permite el tratamiento de las enfermedades y trastornos neuropsiquiátricos (McMahon, 2016), ralentizar el deterioro cognitivo y envejecimiento celular (Stroustrup y otros, 2016) y al mismo tiempo la posibilidad de mejorar las funciones cognitivas de la población saludable en contextos no-clínicos (Sahakian y LaBuzetta, 2015). Aunque la investigación básica se dirige a intentar curar las enfermedades y a tratar las dolencias, la curiosidad humana y el deseo de perfeccionamiento nos da esperanzas de poder algún día mejorar nuestra condición «normal» aplicando estas tecnologías biomédicas para hacer del mundo un lugar mejor. No obstante, el desarrollo biotecnológico y la aplicación de tecnologías que pueden transformar de manera radical nuestra naturaleza no están exentos de debate ético y jurídico por su impacto sociocultural.

De hecho, la biomejora humana es uno de los temas que mayor interés está atrayendo en la reciente discusión y debate en bioética y neuroética. La imagen de abajo muestra con el uso de la herramienta Google Ngram el número de publicaciones (en formato libro) que se han editado desde 1800 hasta 2008 y que tratan el tema de la biomejora humana. Desde principios del siglo XXI el número de publicaciones sobre mejora humana se dispara con un incremento del $120 \%$.

\section{Google Books Ngram Viewer}

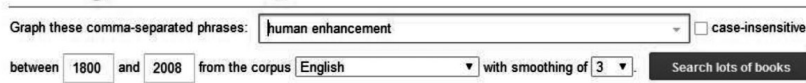

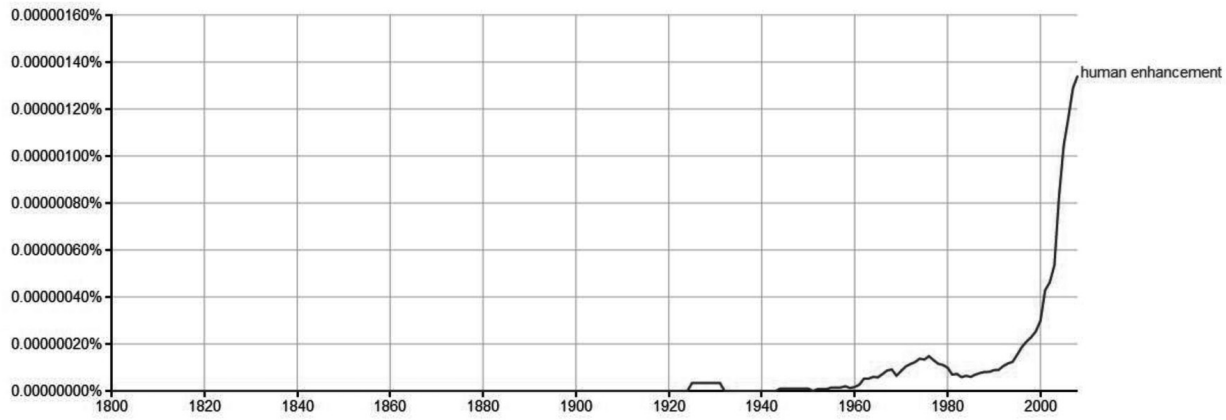

Figura 1

Número de publicaciones desde 1800 hasta 2008 sobre el tema de la biomejora humana 
El informe de la Comisión Presidencial para el Estudio de Asuntos de Bioética de los EE.UU., titulado Beyond Therapy (Kass, 2003b), presentó los resultados de su estudio sobre el uso de intervenciones biomédicas para mejorar procesos cognitivos como la memoria, inteligencia o modulación de estados afectivos, así como la mejora del rendimiento físico en atletas y la extensión de la vida.

Aunque el tono general de este famoso informe pone objeciones a la perfección tecnológica e insta a salvaguardar y conservar la actual naturaleza humana que a día de hoy dispongamos del conocimiento y la tecnología para modificar nuestra naturaleza hace imposible evitar reflexionar qué argumentos a favor o en contra existen para necesitar la biomejora humana.

Dentro de la biomejora humana se puede diferenciar la «biomejora moral» como un tipo de mejora específica cuyas implicaciones son muy profundas. La moralidad es un elemento central de nuestra naturaleza y personalidad y de cómo entendamos qué es correcto o incorrecto en nuestras decisiones depende en gran medida la buena marcha de nuestras sociedades, la supervivencia de nuestra especie y la sostenibilidad del planeta. Sin embargo, como se verá en la sección 3 acerca de la anatomía de la moral articulada por DeGrazia definir la biomejora moral es problemático. Este escrito no tiene como proposito establecer criterios para una definición óptima de biomejora moral, pero se puede entender que la biomejora moral hace referencia al uso de tecnologías que mejoran, alteran o modifican la conducta o psicología moral.

Es este sentido amplio de biomejora moral el que se usa implícitamente a lo largo de este escrito, aunque hay múltiples razones para una mayor clarificación conceptual del término (Raus y otros, 2014). Algunas de estas razones son:

1) biomejora moral es un concepto ambiguo, muy mal definido que dificulta un debate constructivo

2) confusión entre intervenciones especificas y experimentos mentales.

3) cómo se relaciona la intervención con la mejora moral

4) se necesita diferenciar entre el tratamiento de patologías morales y mejoras morales (aunque la distinción entre mejora y tratamiento para algunos autores, por ejemplo Julian Savulescu, no conlleva ninguna diferencia moral y hay que eliminar dicha distinción entre tratamiento y mejora)

Un buen punto de partida para clarificar el término de biomejora moral es tomar el enfoque "welfarista de mejora» (Savulescu y otros, 2011). A nivel internacional dos de los defensores más conocidos de la biomejora moral son Julian Savulescu e Ingmar Persson, ambos del Oxford-Uehiro Centre for Practical Ethics de la Universidad de Oxford, que en 2008 publicaron conjuntamente un libro en el que urgen a la modificación artificial de nuestra psicología moral natural para poder hacer frente a los grandes problemas (cambio climático, guerras, riesgos existenciales...) que amenazan el bienestar y el futuro sostenible de nuestra especie y planeta.

En nuestro país el proyecto de investigación «Ética y política de los avances biomédicos en mejora humana» (ref. FFI2012-32565) dirigido por Francisco D. Lara Sánchez y en el que colaboraron diversos investigadores como Javier Rodríguez Alcázar, Miguel Moreno Muñoz, Paloma García Díaz, Olga Campos Serena, Blanca Rodríguez López, Julian Savulescu, Guy Kahane, Bennett Foddy 
y Andy Miah; ha pretendido durante su tiempo de ejecución a través de congresos y seminarios, publicaciones y otras actividades examinar los distintos aspectos científico-técnicos, filosóficos (epistemológicos, antropológicos, éticos) y el impacto socio-cultural de las tecnologías de biomejora. Tecnologías para mejorar la capacidad moral incluyen la ingeniería genética, intervenciones farmacéuticas (oxitocina, modafinil, ritalin...) y que recientemente este armamentarium de herramientas se ha extendido para incluir el uso de la Inteligencia Artificial como apoyo computacional para tomar la mejor decisión moral (Savulescu y Maslen, 2015; Borenstein y Arkin, 2016; Conitzer et al., 2017).

El presente artículo considera la cuestión de la permisibilidad ética de las tecnologías de biomejora moral humana, considerando las tecnologías en su sentido más amplio, teniendo en cuenta los argumentos de dos posiciones contrapuestas. Por un lado, la posición de David DeGracia (2014) que defiende la deseabilidad de las tecnologías de biomejora moral humana y, por otro lado, la posición de John Harris (2011) que argumenta en contra de las tecnologías de biomejora moral humana porque cree que suponen una amenaza para la libertad. Se concluye que no hay nada intrínsecamente malo en las tecnologías de biomejora moral y si se cumplen principios bioéticos básicos, es éticamente permisible como veremos en la sección 4 .

Actualmente, existen en el mercado medicamentos o tratamientos farmacológicos que producen una mejora sustancial en la población paciente de la memoria (Baro y otros, 2003), atención (Turner y otros, 2004), planificación, control del comportamiento impulsivo (Voon y otros 2015), memoria de trabajo (Mehta y otros, 2004), y hasta incluso habilidades matemáticas (Cohen Kadosh y otros, 2010). Por supuesto, otras muchas vías de mejora humana están en fase de prueba de concepto, fase de ensayo o por desarrollarse (MIT Technoloy Review , 2016).

Una clasificación genérica y amplia, pero útil, aunque pueden darse otros criterios de clasificación; es agrupar las tecnologías de biomejora moral humana en tres grandes categorías:

- Consumibles

- Intervenciones

- Servicios

Los consumibles hacen referencia a todo fármaco, medicación, gragea etc. cuya ingesta, inoculación o cualquier otra vía de absorción y metabolización por el organismo puede alterar o modificar algún componente o aspecto de nuestra cognición y comportamiento moral. Ejemplos son la «clozapina» nombre comercial para un antipsicótico atípico que es un inhibidor de la recaptación de la serotonina que reduce las conductas agresivas y violentas fomentando la conducta prosocial (Crockett y otros, 2015).

Por intervenciones, se hace referencia al diseño del entorno para producir un cambio en la conducta a veces mucho más efectivo que cambiar la propia mente de las personas o cualquier otro aspecto interno. Una idea traída de la economía del comportamiento que se incluye en la categoría de intervenciones es la «arquitectura de la elección» (Thaler y otros, 2010). Una "arquitectura de elección» es todo aspecto del entorno que impele a comportarnos de una determinada forma, sin coaccionarnos, ni impedir otras alternativas disponibles, sin cambiar los incentivos económicos y que además incrementa nuestro bienestar subjetivo. 
Un ejemplo tomado prestado del libro Nudge de Thaler y Sunstein (2009, 5-6) muestra la «arquitectura de elección» en acción para la lucha contra la obesidad. Colocando en los expositores de los restaurantes o comedores aquellos platos dietéticamente más saludables en una posición más visible a la altura de los ojos y relegando a una zona menos visible aquellos platos que contienen mayores cantidades de grasas y azúcares, malos para nuestra salud, se puede reducir la incidencia de la obesidad y todo ello sin tener porque prohibir la «comida basura».

La idea de una «arquitectura de elección» se puede extrapolar a la dimensión moral. Los ojos son estímulos biológicamente relevantes por su importancia en la comunicación social. Colocar unos ojos esquemáticos en los pasillos de un supermercado podría reducir el número de hurtos, al representar esos ojos esquemáticos «alguien que nos vigila». El concepto de nudge o «arquitectura de elección» podría ser una forma de mejora moral no invasiva y muy eficaz.

Finalmente, dentro de la categoría de servicios se puede hacer referencia a cualquier trabajo de una entidad pública o privada que permita ofrecer un servició biomédico que modifique o altere algún aspecto de nuestra moral. Por ejemplo, una clínica que seleccione embriones que contengan en su genoma genes responsables de la expresión de rasgos psicológicos que propicien la empatía o cualquier otra conducta prosocial.

Los consumibles serían internos, las intervenciones externas y los servicios pueden ser ambos. Por interno y/o externo, como ejes, se hace referencia al mecanismo de acción de la biomejora: desde fuera (externo, ejemplo una política pública) o desde dentro (interno, ejemplo pastilla).

Las tecnologías de biomejora moral humana (habría, posiblemente, otras consideraciones éticas al tratar la biomejora moral de otros primates no-humanos $\mathrm{u}$ otros animales), de ahora en adelante $\mathrm{BM}(\mathrm{H})$ con o sin la $\mathrm{H}$ de humana, independientemente de cómo las clasifiquemos, han copado los recientes debates bioéticos dentro de la reflexión en torno a la empresa general de la mejora humana por varias razones (Savulescu y Bostrom, 2013). Ponen en relación varias áreas de estudio como la neurociencia y disciplinas afines dentro de las ciencias de la mente y del cerebro y una de las áreas más importantes dentro de la filosofía: la ética. Y quizá la razón más convincente, por las implicaciones individuales y sociales de la posibilidad de inducir y/o alterar comportamientos morales.

Como toda tecnología que potencialmente puede modificar radicalmente nuestra forma de vivir y de trabajar hay opiniones mutuamente excluyentes. Hay personas que se oponen de plano a las tecnologías de BM por temor. Hay críticos que temen que las tecnologías de BM amenazan nuestra libertad, nuestra naturaleza auténtica y originaria, nuestro sentido del yo, o simplemente por las consecuencias no previsibles que para la sociedad tendrían las tecnologías de BM (Sandel, 2004).

En las siguientes páginas, en las secciones 2 y 3, se examinan dos de estas posturas contrapuestas. Por un lado, la defendida por Harris (2011) que afirma que la BM supone una amenaza para la libertad humana y, por otro lado, la defendida por DeGrazia (2012) donde apoya una tesis de la deseabilidad de la BM. En la sección 4 asumiendo los argumentos en favor de la deseabilidad de las tecnologías de BM de DeGrazia se apoya una permisibilidad ética por la conexión lógica y causal existente entre las razones coherentes de la deseabilidad y la permisibilidad ética. 
Independientemente de dónde uno se sitúe en el debate bioético en torno a las tecnologías de BMH un ejercicio de prudencia y precaución, así como un análisis del coste y beneficio de toda alteración o modificación de nuestra capacidad moral, es necesario. Para ello, en la sección 5, también se da cuenta de una simple propuesta de guía de uso y regulación de las tecnologías de BM siguiendo el principio de precaución para proteger la salud.

En la sección 6 de discusiones se acepta la ida de que si las tecnologías de BMH cumplen con principios bioéticos básicos de seguridad, efectividad y acceso no se deriva nada intrínsecamente malo en alterar o modificar el comportamiento moral, afirmando la permisibilidad ética de la aplicación de tecnologías de BM.

\section{El cuasi-intelectualismo socrático de Harris y la amenaza a la libertad de la BM}

El intelectualismo moral clásico, el atribuido a Sócrates, sugiere que uno hará lo que es correcto o mejor tan pronto como uno conozca lo que es correcto y mejor. La virtud (e.g. modo de ser, carácter, disposición, habilidad etc.) se reduce a una cuestión de conocimiento intelectual, de ahí el nombre intelectualismo moral y se le añade el adjetivo clásico por ser expuesto por primera vez por Sócrates.

John Harris (2011) considera que «una cosa que podemos decir con confianza es que el conocimiento ético no es ser bueno siendo bueno, sino más bien ser mejor en conocer el bien y entender qué es lo que conduce al bien». Para Harris, el espacio entre conocer el bien y hacer el bien es un espacio enteramente habitado por la libertad (el libre albedrío) y aunque el conocimiento de lo bueno es suficiente, la libertad lo es todo, incluso, la libertad para decidir incorrectamente (equivocarse: «fall»y «fail»).

Harris prosigue arguyendo $(2011,104)$ que «aquellos con la perspicacia, simpatía, entendimiento empático y conocimiento para formar ideas claras de lo que puede conducir al bien, no son necesariamente mejores a la hora de hacer el bien...» Razones hay muchas según Harris, pero una parece ser importante: la akrasia o debilidad de la voluntad.

Tomando la expresión de George Bernard Shaw de virtud como «tentación insuficiente» Harris conecta la akrasia o debilidad de la voluntad con la misma virtud, porque algo que no se ha discutido ampliamente es el hecho, según Harris, de que los mismos rasgos o disposiciones que nos llevan a la maldad o inmoralidad son los mismos que nos conducen a la virtud o a una vida moral.

Dicho de otra forma, si no sabes que eres malo no puedes ser bueno y lo que te permite pasar de ser malo a ser bueno es el conocimiento del bien y, aún más importante, la libertad de errar o equivocarte para aprender de ello. La BM considera Harris, y utiliza ejemplos traídos de Tom Douglas de BM para reducir reacciones emocionales adversas ante miembros de otras razas o la agresión violenta (Douglas, 2008), elimina la posibilidad de hacer cosas inmorales.

El racismo es moralmente malo, pero de acuerdo con Harris una intervención de BM que elimine las emociones negativas que crean el racismo cuando ves a otro distinto a ti, no permite conocer que el racismo es malo experimentando vergüenza $\mathrm{y}$ aprendiendo que los prejuicios que de una u otra forma todos tenemos, no deben de guiar tus acciones. La libertad para hacer cosas inmorales con la BM se ve 
amenazada y por tanto no aprendemos a no hacerlas mediante la prudencia y la razón, sino con una intervención de BM sea cuál sea esta.

Harris $(2011,107)$ sigue exponiendo ejemplos de autores que defienden la BM, como Persson y Savulescu (2008) que aducen que la biomejora cognitiva hace urgente primero la necesidad de BM para que no ocurran catástrofes que pueden ponernos en peligro como especie, o Rees (2004) que sugiere que los desastres que puedan poner en peligro la existencia de la humanidad pueden ser no tanto por maldad, sino por estupidez o incompetencia. No obstante, para todas estas posiciones que defienden la BM, Harris sigue creyendo que la mejor estrategia es dejarnos libres para encontrar las mejores soluciones a nuestros problemas o dejarnos libres para errar. Esta misma posición contraria a la BM, aunque Harris no ve nada malo en la mejora cognitiva porque cree que para ser moral uno ha de ser racional, la sigue manteniendo en su reciente libro (Harris, 2016).

\section{DeGrazia y la deseabilidad de la BM}

Como posición antagónica a la de Harris (2011), DeGrazia (2014) argumenta en contra y defiende la deseabilidad de la BM. DeGrazia $(2014,364)$ comienza reconociendo el creciente interés por «mejorar» características humanas en contextos biomédicos. Artículos académicos, libros etc. han aparecido en los últimos tiempos tratando y examinando temas como el doping en el deporte, el mejoramiento del estado de ánimo y cognición por medios farmacológicos, genéticos... (Véase, la imagen de la introducción).

Estas intervenciones biomédicas son mucho más complejas que los «mejoramientos» tradicionales como la educación o cualquier otro tipo de entrenamiento y aprendizaje. Aún más reciente es la emergencia en la literatura e investigación de la BM. Intervenciones destinadas a mejorar nuestras capacidades morales como nuestras disposiciones para la simpatía, equidad etc. DeGrazia nos ofrece algunos ejemplos de intervenciones de BM como:

- Inhibidores selectivos de la recaptación de la serotonina como un medio para reducir la agresividad (Crockett y otros, 2015, Crockett y otros, 2010)

- Propanolol como un medio para reducir los estereotipos y prejuicios racistas (Terbeck y otros, 2012).

- Estimulación cerebral profunda como medio para reducir la agresividad (Franzini y otros, 2005).

- Neruofeedback para tratamientos de trastornos de personalidad (Sitaram y otros, 2007).

- Selección de embriones que contengan genes que codifiquen rasgos del comportamiento que conduzcan a la prosocialidad (Eley y otros, 1999).

Estos y otros ejemplos son según DeGrazia intervenciones que podemos llamar colectivamente de BM. La BM ha de considerarse como una posibilidad seria para hacer frente a los riesgos y amenazas globales que ponen en riesgo a la humanidad. La tecnología y el conocimiento ya está disponible y los daños e injusticias que es capaz de generar el ser humano por sus déficits morales convierten a la BM como una posibilidad a tener en cuenta para hacer del mundo un lugar mejor. 
¿Por qué considerar la BM humana una posibilidad seria? DeGrazia presenta una larga serie de ejemplos de comportamientos inmorales que de una u otra forma se atajarían con la ayuda de la BM. Ejemplos de comportamientos inmorales con consecuencias devastadoras y grandes riesgos son los genocidios (Ruanda, Bosnia...), la aniquilación nuclear, las pandemias, el bioterrorismo, ciberterrorismo, cambio climático...

Todos y cada uno de estos ejemplos de problemas globales se pueden atribuir a déficits morales en los individuos que tienen consecuencias para el planeta y que potencialmente pueden ejercer un daño masivo al ser humano etc. porque en buena medida se magnifican con el desarrollo de la tecnología situándonos en un escenario donde un número limitado de individuos pueden tener el poder de infligir mucho daño a gran escala (Persson y Savulescu, 2008).

Como dice DeGrazia el statu quo moral del ser humano es problemático y algunos consideran que mantenerlo de esta manera es intolerable. Hasta ahora hemos dispuesto de medidas de mejora moral tradicional: educación, socialización, diseño de políticas públicas... pero apunta DeGrazia que estas son incapaces de producir una mejora significativa y por esta razón la BM merece nuestra consideración sin preconcepciones.

Para poder alterar, modificar o mejorar nuestras capacidades morales DeGrazia articula una anatomía de la mejora moral muy interesante que puede servir de esquema aplicable para discernir el tipo de tecnología de BM y qué componente de la moral alteraría. Esta anatomía de la moral diferencia tres elementos: motivación moral, cognición moral y comportamiento moral. Esta distinción es muy pertinente e importante porque ha de tenerse en cuenta a la hora de salir de un discurso y debate sobre las tecnologías de BM eminentemente especulativo y maniqueo entre posiciones dicotomizadas y simples (bioconservadurismo Vs posthumanismo). Las tecnologías de BM pretenden alterar, modificar, o mejorar un sistema biológico complejo como el de nuestra psicología moral natural con mecanismos y submecanismos sobre los que todavía no hay un consenso acerca de cuáles están implicados (Shook, 2012; Greene, 2015) y el debate ético sobre la mejora humana se concentra cada vez más en dominios muy específicos, lo que requiere que la reflexión ética sea de un mayor rigor analítico (Rodríguez y otros, 2015). La posibilidad práctica y el estado del arte de la investigación nos harán tener una visión mucho más pragmática y realista de las tecnologías de BM.

Pero no debemos olvidar que hay muy pocos rasgos o aspectos del desarrollo humano que son poligénicos, es decir, que un rasgo o aspecto del desarrollo humano está controlado por la acción de múltiples genes. Esto conlleva una explosión combinatoria. De nuestros aproximadamente 25.000 genes, cada uno de estos genes interactúan de muchas maneras entre si y entender en gran detalle cómo interactúan es extremadamente difícil. Y esta explosión combinatoria, o poligenia, se extrapola al desarrollo cerebral y por ende a la psicología moral. ¿Cómo podemos intervenir para mejorar la capacidad moral humana cuando esta depende de una vasta red interacciones a nivel molecular, celular, sistémico y hasta cultural? Sin tener en cuenta factores epigenéticos que hacen que se expresen o supriman ciertos genes por influencia del entorno. Por ejemplo, modificar o alterar la predisposición al comportamiento altruista a través de los niveles de oxitocina que a su vez inducen en una mayor respuesta empática y por tanto hacen de una 
persona más altruista puede tener efectos indirectos cascada en otros subsistemas que sean poco beneficiosos. Uno de ellos que al incrementar el altruismo mediando los niveles de oxitocina es muy probable que una persona tienda a favorecer a miembros de su endogrupo (DeDreu et al., 2011).

No obstante, siguiendo con la distinción de la anatomía de la moral de DeGrazia, el autor se disculpa de que esta anatomía de la mejora moral pueda ser una simplificación (los internalistas o los emotivistas en teoría moral pueden no separar la motivación de la cognición porque a la hora de decidir si algo es correcto o no en un juicio moral, uno ha de tener una motivación o emoción para ello) pero de todas formas puede facilitar la discusión sobre los tipos de la BM y qué componente de la moral altera.

Ante la cuestión: ¿qué componente de la moral alterar?, otro de los principales retos es el del contenido. La sociedad sostiene una pluralidad de visiones de lo que la moral requiere. La gente tiene diferentes opiniones sobre lo que ha de ser una vida moral. Algunos, dice DeGrazia $(2014,363)$, pueden considerar que la moral se ha de adherir a valores políticos conservadores (primar la responsabilidad individual, tradiciones sociales honorables, defensa de la comunidad y la religión propias) pero otros pueden considerar que la moral se ha de adherir a valores políticos progresistas (compasión por aquellos que no tienen las necesidades básicas cubiertas, intervención del estado para garantizar el bienestar de los ciudadanos, ser inclusivos con otros pueblos etc.) Entre los teóricos de la ética hay deontologistas, utilitaristas, teóricos de la virtud que no solo tienen diferencias meramente teóricas, sino que sus planteamientos se traducen en diferentes visiones normativas sobre asuntos prácticos como la eutanasia, aborto, cuidado animal, drogas, relación iglesia-estado etc.

Ante esta gran diversidad sobre qué ha de ser la moral, qué alteración o cambio ha de verse como mejora moral, se plantea un problema no solo para las tecnologías de BM, sino también para métodos de mejora moral tradicionales como la educación. No hay un criterio para determinar qué es correcto y por tanto los padres no pueden estar seguros de si están enseñando a ser morales a sus hijos o instruyendo e inculcando ciertos valores.

Tanto para las personas individuales como para el estado que algún día tenga que subsidiar tecnologías de BM este es un gran reto: qué es lo que se supone que será una mejora en términos del contenido de la moral. DeGrazia (2014, 364) sugiere que toda BM debe representar puntos de consenso superpuestos entre perspectivas morales rivales y razonables. Para entender qué es razonable De Grazia pone ejemplos de contraste. El libertarismo, el socialismo, el conservadurismo son razonables mientras que el nazismo, el apartheid, los talibanes, no lo son. La razonabilidad puede ser laxa dejando abierta la posibilidad de que las decisiones de los padres sobre qué moral hacer desarrollar en sus hijos sea debatible y por supuesto toda acción política de BM debe ser razonable desde cualquier visión de la moral.

Acertada o no la respuesta para hacer frente al reto del pluralismo moral, buscar la razonabilidad en nuestras sociedades, DeGrazia pasa a defender la deseabilidad de la BM. Y presenta una situación idealizada. Imagina que la BM es segura y eficaz que no son caras y que el estado las financia. Imagina que las tradicionales formas de mejora moral se demuestran ineficaces. Imagina también que no hay 
que temer las consecuencias a largo plazo de la BM en la sociedad (diferencias entre los mejorados y los no mejorados). Formuladas todas estas idealizaciones lo que queda es que no hay nada inherentemente malo en la BM. Argumentos en contra de la BM señalan que la BM es antinatural y que no reconocen el regalo de nuestra vida (Sandel, 2004) o que ponen en peligro nuestra identidad personal. Harris $(2011,104)$ dice «el espacio entre conocer el bien y hacer el bien es un espacio enteramente habitado por la libertad (el libre albedrío). El conocimiento de lo bueno es suficiente, la libertad lo es todo».

Para replicar a la objeción de Harris es preciso contestar a dos preguntas, nos dice DeGrazia: ¿Hasta qué punto la BM amenaza la libertad? y ¿cuán importante es la libertad incluida la libertad para errar? Una concepción general del libre albedrío sostiene que los agentes humanos son «motores inmóviles» que son capaces de actuar libremente y de introducir causalidad en el orden natural de las causas y eventos sin estar ellos mismos sujetos al orden natural. Esta concepción dice que no podemos ser libres si algo o alguien causa o determina lo que elegimos o hacemos.

El compatibilismo es una posición en el debate sobre la libertad que permite esta concepción del libre albedrío o libertad coexistiendo con un orden causal universal determinista. Esta posición es humeana en el sentido de que somos libres siempre que actuemos de acuerdo a nuestra voluntad que determina lo que hacemos y queremos. Por ejemplo, dice DeGrazia $(2014,365)$ imagina que me abstengo de comer tras cenar no porque nadie me obligue o porque tenga anorexia, sino porque quiero mantener una dieta sana y abstenerme de comer tras cenar es un medio para conseguir este objetivo. ¿Hay alguna razón para pensar que la BM supone alguna amenaza a mi libertad bajo esta concepción de libertad? DeGrazia claramente dice que no.

Como resultado de una BM yo podría tener una fuerza de voluntad mayor y por tanto ser menos vulnerable a debilidades volitivas, menos inclinado a actuar bajo impulsos violentos, dispuesto a actuar altruísticamente y también inclinado a actuar justamente. Pero el escéptico puede responder que lo que verdaderamente te hace libre es poder haber hecho otra cosa á la Frankfurt. La BM puede generarte una fuerte motivación o fuerza de voluntad e impedirte comer después de cenar, pero ¿podrías haber hecho otra cosa?, es decir, ¿podrías haber cenado? DeGrazia dice que sí. Bajo esta concepción de libertad o libre albedrío actuar libremente no es estar libre de una influencia causal y aunque haya una presión causal externa uno no es un sujeto pasivo de esta influencia, sino que uno la recibe de buen grado.

En esta concepción compatibilista de la libertad actuar libremente requiere no actuar con perfecta independencia de influencias causales-que es imposible en esta concepción-sino la correcta relación entre nuestras preferencias y acciones como la ausencia de ciertas condiciones que revierten la libertad. Como dice DeGrazia no hay una amenaza a la libertad por parte de la BM si esta actúa en la motivación del sujeto.

Sobre el valor de la libertad en la moralidad DeGrazia considera que es importante, pero que este valor no elimina todo lo "otro" que puede tener importancia. Es decir, los grados de libertad se han de poner en una balanza con otros valores igual de importantes. Imagina, dice DeGrazia, que tenemos una forma de medir lo que la $\mathrm{BM}$ reduce de libertad y que reduce de libertad un 25\%. Pero imagina también que las tecnologías de BM reducen las guerras, el hambre y todo el mundo tiene acceso 
a las necesidades básicas. DeGrazia dice que estaría de acuerdo en aceptar ese 25\% de reducción de libertad por todas las consecuencias positivas que se derivarían de la BM. Ahora imagina, siguiendo a DeGrazia, que una tecnología concreta de BM pero no otras reduce en un $100 \%$ la libertad, en concreto un chip implantado en el cerebro que se utiliza para impedir que agresores sexuales cometan abusos sobre niños. Esto robaría el 100\% de la libertad del agente con respecto a la agresión hacia los niños dejándole actuar libremente en todas las demás facetas de su vida sin ningún tipo de efecto secundario. De acuerdo con DeGrazia esta perdida de libertad no es una gran pérdida sobre todo si lo comparamos con la pérdida de libertad que se deriva del encarcelamiento de alguien que actúa así cometiendo abusos de niños. La libertad es un valor importante, pero cuando lo comparamos con otros no tiene porque ser siempre el que prime.

Otros autores también han criticado la posición de Harris sosteniendo que la BM no tiene porque reducir la libertad porque de la misma manera que una persona virtuosa o moralmente experta no es menos libre que un «malhechor», el uso de tecnologías de BM no supone una gran diferencia (Diéguez y Véliz, 2017).

\section{De la deseabilidad de las tecnologías de BM a La PERmisibilidad Ética de la BM}

Los argumentos de DeGrazia (2014), expuestos más arriba, muestran la deseabilidad de la aplicación de tecnologías de BM como una alternativa a tomarse muy en serio para hacer frente a los grandes problemas globales que ponen en riesgo nuestra existencia como especie. Y por supuesto, no tienen por qué suponer una amenaza a la libertad si se concibe la idea de libertad desde una perspectiva compatibilista. Por otra parte, no hay nada inherentemente malo en la aplicación de tecnologías de BM, concluye DeGrazia, y además estas pueden acabar con el statu quo moral problemático (guerras, conflictos, efectos del cambio climático...) que tiene la humanidad y que los métodos tradicionales de mejora moral como la educación, socialización etc. no consigue erradicar. Este mismo argumento ha sido utilizado originariamente por Persson y Savulescu (2008) para defender la idea de que necesitamos la mejora tecnológica de la moral.

Desde esta perspectiva las evidencias apuntan a que nuestra naturaleza biológica es deficitaria y los comportamientos inmorales no se pueden eliminar exclusivamente con la educación. Nuestra esperanza para poder evitar un mal mayor o un gran desastre, en la medida en que muchos de los problemas con los que nos enfrentamos como especie son causados por déficits morales a nivel individual, es recurrir al uso de tecnologías de BM (Douglas, 2008). Pero, intuitivamente, se piensa que la deseabilidad no implica la permisibilidad ética. Los riesgos son latentes en el uso y aplicación de tecnologías de BM. El uso de tecnologías de BM puede reducir la criminalidad, incrementar el comportamiento prosocial y la aversión a causar daños o acabar con los grandes problemas globales, pero pueden conducir a consecuencias no previstas y efectos adversos no deseados (Brooks, 2012). Esto significa que algo que sea deseable, no tiene porqué ser permisible éticamente.

Sin embargo, se entiende que si las premisas (razones) de la deseabilidad de las tecnologías de BM son consistentes y coherentes — necesidad de las tecnologías de BM para evitar la naturaleza humana deficitaria y la posibilidad de grandes 
desastres - puede haber una «conexión lógica» con la permisibilidad ética de la BM. Este es el argumento de la «conexión lógica» según el cual hay un vínculo lógico entre la deseabilidad y la permisibilidad. Las descripciones de la deseabilidad (deseos, creencias) justifican la permisibilidad de la acción. Argumentamos a favor de una conexión entre la deseabilidad y la permisibilidad. La deseabilidad y la permisibilidad están íntimamente ligadas. Desde la razón práctica tenemos el siguiente silogismo:

\section{$P 1$ un sujeto $S$ hace A porque desea $D$ y cree que hacer A le permitirá conseguir D.}

Si la valoración moral de D y A es coherente y no viola ningún principio moral, tener el deseo D justifica hacer A y, por consiguiente, A se convierte ceteris paribus en permisible.

Lo deseable es permisible en tanto y cuanto lo deseable tiene un contenido (par creencia-deseo) que es valioso e implica lógicamente su permisibilidad. Es decir, las razones que hacen que algo sea deseable explican conceptualmente su permisibilidad. Si el fin que se persigue con la aplicación de tecnologías de BM es valorable moralmente hablando, no hay contradicción lógica en aceptar su permisibilidad ética. Y de igual modo, aunque es independiente, puede haber una «conexión causal». Lo deseable es permisible en tanto y cuanto lo deseable tiene un contenido (par creencia-deseo) que causa la permisibilidad. Es decir, las razones que hacen que algo sea deseable explican causalmente su permisibilidad. Si el fin que se persigue con la aplicación de tecnologías de BM es valorable moralmente hablando, las razones de aceptar la deseabilidad de dichas tecnologías causan la permisibilidad de la BM. Es verdad que los riesgos y/o costes no se pueden eliminar, pero el fin y objetivo que se persigue compensa. La segunda premisa es:

\section{$\boldsymbol{P 2}$ si deseas $D$ y no hay objeción moral a $D$ entonces es propio que hagas A para conseguir D.}

Este principio o relacionados han sido defendidos con distintos argumentos por varios filósofos en el campo de la epistemología (Hawthorne y Stanley, 2008) y lo que intentan señalar es que el carácter apropiado de una acción en la economía epistémica de un agente está ligado a sus intereses prácticos, concluyendo:

\section{C si y solo si deseas D entonces es apropiado tratar D como «razón» para actuar}

No sabemos si necesariamente conlleva un principio epistémico universal, pero en nuestro comportamiento de sentido común y popular, habitualmente atribuimos esta conexión. Por ejemplo, imagina la siguiente situación: «Él desea ir a casa y sabe que el bus llega en cinco minutos, por qué todavía sigue en la oficina» Que la gente ordinaria juzgue ciertas conductas bajo una relación entre la deseabilidad y la permisibilidad para actuar sobre la base de esos deseos en una conexión estricta, nos da que pensar que ha de existir un principio entre la deseabilidad-permisibilidad. En la situación imaginada la persona que comenta que él desea irse a casa, pero que todavía sigue en la oficina y se pregunta por qué no ha salido de la oficina, entiende una relación clara entre la deseabilidad y la permisibilidad: desear ir a casa conlleva 
la permisibilidad de los intereses prácticos de acción. Solo un principio que conecte deseabilidad con permisibilidad puede dar coherencia a esta acción.

La relación entre la deseabilidad y la permisibilidad es indirecta, pero fuertemente conectada y está mediada por considerar y tratar la relación como verdadera. Desear D, presupone tratar D como verdadera en el razonamiento conducente a la acción.

Dicho con otras palabras, el argumento de la «conexión lógica» entre la deseabilidad de las tecnologías de BM y la permisibilidad ética de la BM conlleva que se siga lógicamente, con necesidad, el uno del otro. Si una acción no entraña conflicto alguno con principios y valores éticos, sus razones son consistentes y coherentes, entonces es permisible éticamente. Las tecnologías de BM son deseables, porque pueden acabar con los grandes problemas de la humanidad, luego las tecnologías de BM son permisibles éticamente hablando.

\section{REgulación y GOBERnANZA DE LAS TECNOLOGías DE BM}

Los argumentos de DeGrazia a favor de la deseabilidad de la BM y en contra de que las tecnologías de BM suponen una amenaza para la libertad son muy razonables, pero suponen situaciones ideales donde principios bioéticos básicos se cumplen o se dan por sentado como, por ejemplo, los principios de seguridad, eficacia, y acceso universal de las tecnologías de BM. Estos principios regulan y prohíben intervenciones e investigaciones biomédicas en seres humanos que puedan producir un daño o riesgos innecesarios tal y como se contempla en las legislaciones y la práctica científica estándar. No obstante, si estos principios se cumplen no hay nada malo per se en la deseabilidad de la BM. Una forma eficaz para conseguir que estos principios se cumplan es regular y controlar de la forma más liberal posible las tecnologías de BM.

En la actualidad no hay un marco político regulatorio sobre tecnologías de biomejora, sean morales o no, a nivel europeo y las iniciativas que se han llevado a cabo no están interconectadas (Coenen y otros, 2011). La UE reaccionó a ciertos debates sobre la biomejora humana en el contexto de la nanotecnología emitiendo un informe final (EU HLEG FNTW 2004) que criticaba la biomejora humana advirtiendo el grupo de expertos que firmaba el informe sobre la posible malversación de fondos públicos. A este informe le siguió una serie actividades de investigación financiada por la UE sobre el tema de la biomejora (Coenen y otros, 2011). El Grupo Europeo de Ética de la Ciencia y de las Nuevas Tecnologías (EGE) también ha analizado la nanomedicina en su opinión número 20. El grupo sugiere que mantener la distinción entre intervenciones terapéuticas y de mejora es importante sobre todo en lo que respecta a la financiación europea, primando las intervenciones terapéuticas. El grupo ha identificado ciertos implantes en el cerebro sobre los que hay que tener especial precaución y su regulación es necesaria porque son implantes que pueden cambiar funciones psíquicas o que pueden tener un uso de manipulación y vigilancia (EGE, 2005).

Declaraciones políticas oficiales de la UE sobre biomejora son escasas y dados los diferentes valores de los estados miembro hay una falta de marco regulativo coherente que pueda hacer frente al control de la biomejora humana. Coenen y 
otros (2009) recibieron el encargo como miembros del ETAG (European Technology Assesment Group) de un estudio sobre la mejora humana y las posibles estrategias para tratar la mejora humana en el contexto de la Unión Europea identificando una posición pro-mejora razonada, otra posición restrictiva razonada y otra de estudio caso por caso. Cinco son las opciones que se pueden pensar sobre la biomejora humana de acuerdo con Coenen y otros $(2011,2009)$ :

1) una prohibición total de las tecnologías de biomejora humana que alteren la naturaleza humana

2) una liberación de las tecnologías de biomejora humana

3) un enfoque protecnología de biomejora humana razonado

4) un enfoque restrictivo razonado

5) un análisis casuístico (caso por caso)

De acuerdo con Coenen y otros (2011) las dos primeras medidas son poco realistas en un contexto de la UE, pero las otras tres opciones que quedan están sujetas a la primacía del principio de precaución. Un principio que recientemente está siendo criticado porque paraliza la investigación y el avance de la ciencia. En concreto, Steven Pinker (2015) en un reciente artículo declara de una forma un tanto provocativa que el único objetivo que debe tener la bioética es "quitarse del medio» para que la investigación biotecnológica se desarrolle sin impedimentos. Esta boutade de Pinker se puede malinterpretar muy fácilmente. El verdadero mensaje de Pinker es criticar las cortapisas que supone el principio de precaución para el avance biomédico. Pinker sugiere que la excesiva argumentación bioética (siendo uno de sus resultados el principio de precaución) está impidiendo que los frutos de la investigación biotecnológica se traduzcan en revolucionarios tratamientos.

La crítica de Pinker a un cierto tipo de discurso bioético es sensata. Pero el verdadero problema es encontrar el punto de equilibrio entre los juicios de inaceptabilidad ética categóricos (moratorias o el recurso al principio de precaución para prevenir la investigación con riesgos no identificados en la biomedicina) y el principio de proacción. Porque tanto un uso abusivo del principio de precaución limita el avance de la investigación con potenciales beneficios como es inconsciente aplicar un principio de proacción sin revisión ética.

No siempre existen las salvaguardas éticas en la investigación y ante ello se debe pronunciar la filosofía y la ética como reconoce el bioético Julian Savulescu (2015) al hacer una recensión del polémico artículo de Pinker, aunque en última instancia Savulescu está de acuerdo con el mensaje de Pinker: cierta forma de hacer bioética obstruye y paraliza la investigación. Por ello, la solución es encontrar el punto de equilibrio entre el principio de precaución y el principio de proacción. El equilibrio es un principio de precaución razonable y sensato. Seguir haciendo buena bioética sin prejuicios, objetiva y científica y no ese tipo de bioética moralista y moralizante cargada de doctrinas no basadas en la evidencia.

La propuesta de Coenen y otros (2011) para un modelo de gobernanza de la mejora humana es que este ha de requerir un debate público y abierto a la participación que articule las distintas posiciones normativas sobre el tema.

1) organizar una evaluación del impacto de la biomejora humana

2) identificar necesidades de investigación 
3) definir los límites dentro de los cuales cada país puede regular la biomejora humana

4) prevenir efectos no deseados y desigualdades a nivel europeo

5) abrir un serio debate sobre el tema.

Estos cinco pasos son una guía sensible sobre el mejor modo de regular y controlar las tecnologías de biomejora y, en particular, las tecnologías de BM; fomentando el cumplimiento de principios bioéticos básicos (seguridad, eficacia y accesibilidad).

\section{Discusiones}

En este escrito se han considerado los retos éticos de las tecnologías de BM desde las perspectivas contrapuestas de dos autores: DeGrazia (2014) y Harris (2011). Por un lado, DeGrazia considera que no hay nada inherentemente malo en la deseabilidad de la BM y, por otro lado, Harris sostiene que la BM supone una amenaza a la libertad. Valorando los argumentos de DeGrazia se concluye que si los principios tradicionales de la bioética como la seguridad, efectividad y universalidad se cumplen no hay nada malo en la biomejora moral per se. De dicha deseabilidad se sigue una permisibilidad ética y para conseguir salvaguardar dichos principios bioéticos, la aplicación de un principio de precaución es crucial. Pero este principio de precaución ha de ser aplicado de una forma razonada y sensata para que la bioética no se convierta en la cortapisa de la investigación científica que trunque las promesas de revolucionarios tratamientos, sino que acompañe la investigación biotecnológica con un control razonado y equilibrado.

\section{BibLIOGRAFÍA}

Barco A. (y otros) (2016). «CREB, memory enhancement and the treatment of memory disorders: promises, pitfalls and prospects». Expert Opin Ther Targets. N ${ }^{\circ}$ 7, (2003), 101114.

Borenstein, J. y Arkin R. Robotic nudges: the ethics of engineering a more socially just human being. Science and Engineering Ethics 22 (1), 31-46.

Brooks T. (2012). «Moral Frankensteins». AJOB Neurosci, no 3(4), 28-30.

Coenen C. (y otros) (2009). «Human Enhancement», IPOL/A/STOA/2007-13, PE 417.483. Brussels: European Parliament.

Coenen C. (y otros) (2010). The Politics of Human Enhancement and the European Union. En Julian Savulescu, Ruud ter Meulen y Guy Kahane (coords), Enhancing Human Capacities. Oxford: Wiley-Blackwell, pp. 521-535.

Cohen Kadosh R. (y otros) (2010). «Modulating Neuronal Activity Produces Specific and Long-Lasting Changes in Numerical Competence». Current Biology. $\mathrm{n}^{\circ} 22,2016-2020$.

Conitzer V et al. (2017). «Moral Decision Making Frameworks for Artificial Intelligence». Proceedings of the Thirty-First AAAI Conference on Artificial Intelligence (AAAI-17). 48314835.

Crockett M. (y otros) (2015). «Dissociable effects of serotonin and dopamine on the valuation of harm in moral decision-making». Current Biology. $\mathrm{n}^{\circ}$ 25, 1-8.

Crockett M. (y otros) (2010). "Serotonin selectively influences moral judgment and behavior through effects on harm aversion». PNAS. $\mathrm{N}^{\circ} 107,17433-8$. 
De Dreu CK. (2010). The neuropeptide oxytocin regulates parochial altruism in intergroup conflict among humans. Science. 11 328, 1408-11

DeGrazia D. (2014). «Moral enhancement, freedom, and what we (should) value in moral behaviour». Journal of Medical Ethics. $\mathrm{n}^{\circ}$ 40, 361-368.

Diéguez A. y Véliz C. (2017). «Would moral enhancement limit freedom?» Topoi (2017) DOI $10.1007 / \mathrm{s} 11245-017-9466-8$

Douglas T. (2008). «Moral enhancement». J Appl Philos, n 25(3), 228-245.

EGE (European Group on Ethics in Science and New Technologies) (2005). «Ethical Aspects of ICT implants in the human body». Opinion No. 20. Brussels: European Commission. Eley T. (y otros), «Sex differences in the etiology of aggressive and nonaggressive antisocial behavior: results from two twin studies». Child Dev. no 70, (1999), 155-68.

EU, HLEG, FNTW (EU High Level Expert Group) (2004). «Converging Technologies». Brussels: European Commission.

Franzini A. (y otros) (2005). "Stimulation of the posterior hypothalamus for medically intractable impulsive and violent behavior». Stereotact Funct Neurosurg. no 83, 63-6.

Greene, J. (2015). «The rise of moral cognition» Cognition. $\mathrm{n}^{\circ}$ 135, 39-42.

Harris J. (2011). «Moral enhancement and freedom». Bioethics. $\mathrm{n}^{\circ}$ 25, 102-11.

Harris J. (2016). How to be Good: The Possibility of Moral Enhancement. Oxford: Oxford University Press.

Hawthorne, J., \& Stanley, J. (2008). «Knowledge and action». Journal of Philosophy, 105(10), $571-90$

Kass L. (2003b). Beyond Therapy: Biotechnology and The Pursuit of Happiness. Washington, DC: President's Council on Bioethics.

McMahon J. (2016). «Genetic association studies in psychiatry: time for pay-of» The Lancet Psychiatry, S2215-0366.

Mehata M. (y otros) (2004). «Methylphenidate improves working memory and set-shifting in AD/HD: relationships to baseline memory capacity». J Child Psychol Psychiatry, no 45, 293-305.

MIT Technology Review, «10 Breakthrough technologies 2016.» en: MIT Technology Review 2016]https://www.technologyreview.com/lists/technologies/2016/] Recuperado [3 febrero 2016]

Persson I. (y J. Savulescu) (2008). «The perils of Cognitive Enhancement and the Urgent Imperative to Enhance the Moral Character of Humanity». Journal of Applied Philosophy. $\mathrm{n}^{\circ} 25,162-177$.

Pinker S., «The moral imperative for bioethics». en: Boston Globe 2016 [https:// www.bostonglobe.com/opinion/2015/07/31/the-moral-imperative-forbioethics/ JmEkoyzlTAu9oQV76JrK9N/story.html] Recuperado [1 febrero 2016]

Raus, K. (y otros) (2014). «On Defining Moral Enhancement: A Clarificatory Taxonomy.» Neuroethics $n^{\circ} 7(3), 263-273$.

Rees M. (2004). Our Final Century. London: Arrow Books.

Rodriguez B. (y otros) (2015). «Introducción: Bioética y mejora humana». Dilemata, nº 19, $1-5$

Sahakian B. (y J. LaBuzetta) (2015). Bad Moves: How Decision Making Goes Wrong, and the Ethics of Smart Drugs. Oxford University Press.

Sandel M., "The case against perfection» en: The Atlantic Monthly 2004 [http://www. theatlantic.com/magazine/archive/2004/04/the-case-against-perfection/302927/] Recuperado [1 febrero 2016]

Savulescu J. (y otros) (2011). «Well-being and enhancement,» En Julian Savulescu, Ruud ter Meulen y Guy Kahane (coords), Enhancing Human Capacities. Oxford: Wiley-Blackwell, pp 3-18

Savulescu J. (y N. Bostrom) (2013). Human Enhancement. Oxford: Oxford University Press. Savulescu J., «Pinker bioethics: What should we learn?» en Practical Ethics blog 2015 
[http://blog.practicalethics.ox.ac.uk/2015/08/pinker-bioethics-what-should-we-learn/] Recuperado [3 febrero 2016]

Savulescu, J. and H. Maslen (2015). Moral Enhancement and Artificial Intelligence: Moral AI? En Jan Romport, Eva Zackova y Jozef Kelemen (eds). Beyond Artificial Intelligence, Springer, (2016), pp. 79-95

Shook J. (2012). «Neuroethics and the possible types of moral enhancement» AJOB Neurosci, $n^{\circ} 3(4), 3-14$.

Sitaram R. (y otros) (2007). "fMRI brain-computer interface: a tool for neuroscientific research and treatment». Comput Intell Neurosci. n ${ }^{\circ}$ 207. DOI: 10.1155/2007/25487.

Stroustrup N. (y otros) (2016). «The temporal scaling of Caenorhabditis elegans ageing» Nature $\mathrm{n}^{\circ} 530,103-107$.

Terbeck S. (y otros) (2012). «Propranolol reduces implicit negative racial bias». Psychopharmacology (Berl). $\mathrm{n}^{\circ}$ 222(4), 19-24.

Thaler R. H. y Sunstein C. R. (2009). Nudge: Improving Decisions abouth Health, Wealth and Happiness. London: Penguin Books.

Thaler R. (y otros) «Choice Architecture.»en: SSRN 2015 [http://papers.ssrn.com/ sol3/ papers.cfm?abstract_id=1583509]

Turner D. (y otros) (2004). "Modafinil Improves Cognition and Attentional Set Shifting in Patients with Chronic Schizophrenia». Neuropsychopharmacology. n 29, 1363-1373.

Voon V. (y otros) (2015). «Waiting Impulsivity: The Influence of Acute Methylphenidate and Feedback». Int J Neuropsychopharmacology. Doi: 10.1093/ijnp/pyv074. [Epub ahead of print]

Universidad del País Vasco

Aníbal Monasterio Astobiza

anibal.monasterioastobiza@philosophy.ox.ac.uk

[Artículo aprobado para publicación en diciembre de 2016] 